The International Journal Of Engineering And Science (IJES)

|| Volume || 5 || Issue || 11 || Pages || PP 38- 43 || 2016 ||

ISSN (e): $2319-1813$ ISSN (p): $2319-1805$

THE IJES

\title{
Comparative Investigation Ofinter-Satellite Optical Wireless Communication By Using Different Modulation Formats
}

\author{
Dr. Entidhar Mhawes Zghair \\ Middle Technical University, Baghdad, IraqTechnical Instructors Training Institute- Electrical Engineering \\ Department
}

\begin{abstract}
The optical wireless communication systems have got greater popularity in the previous couple of years because of its benefit over conventional radio frequency conversation structures. This paper reviews the effect of the usage of $N R Z, R Z$ and Gaussian pulse generator modulation codecs on the performance of the optical wireless communique (OWC) channel in terms of Quality factor aspect and Minimum BERat bit rate of $10 \mathrm{Gbps}$. It has been watched that NRZ function generator gives better execution for optical wireless association conversely with $R Z$ and Gaussian association for different values of aperture diameters and range.
\end{abstract}

Keywords: Non-Return to zero (NRZ), Inter-satellite optical wireless communication (IsOWC), Q Factor, $B E R$.

Date of Submission: 17 May 2016

$\longrightarrow$ Date of Accepted: 12 November 2016

\section{INTRODUCTION}

Over the long decades, attention continued to OWC restraint, mainly to be used covertly marine applications, [1]. In addition tospace programs such as inter-satellite TV for PCand profound-space hyperlinks. [2]. OWC's It has been spreading rapidly mass market so farrestrained except for IrDA which became a surprisingly a success wireless brief-range transmission solution.[3]. Improvement of modern and effective wireless technologies for a number of transmission links is essential for constructing destiny heterogeneous conversation networks to aid a huge range of service sorts with various patterns and to meet the ever-increasing demands for higher information fees. Variations of OWC ability be potentially utilized in anumerous variety of verbal exchange packages ranging from optical interconnects inside integrated circuits thru outside interconstructing hyperlinks to satellite communications.

The optical wireless communique (OWC) is a verbal exchange era that makes use of line of sight (LOS) direction propagating in free area for transmission of sign/information/information between two favored focuses for media communications or computer networking organizing[4]. The OWC is based on the optical communication with best A distinction that this device does now not use solids as a transmission medium together with optical fiber cable or optical transmission line. OWC is basically followed when Thebodily connection isn't always almost viable due to excessive fee or other consideration [5]. Hence, this technology utilized air as the environment of a transmission, the sensitivity towards atmospheric phenomena is inevitable. Those disturbances will appreciably have an effect on the OWC transmission performances. The climatic turbulences will purpose the fast fluctuation of receiving electricity and subsequently will lessen the device excels. Moreover, the interruption of the laser beam consisting of cook flap can even disturb the verbal exchange channel energy which may be stepped forward by way of the use of amplifier [6]. OWC hyperlink is still taken into consideration, particularly as a new technology, although fiber optic verbal exchange has been widely used in global extensive Telecommunications industry.This work makes a specialty of distinct modulation codec's and their effect on the rate of transmission for LEO ISLs. This paper pursuits to find the most suitable modulation layout for helping excessive speed ISLs. The performance of numerous modulation sorts was investigating the use of the Q-factor and BER..

\section{SYSTEM DESCRIPTION}

To investigate the performance of the optical wireless communication (OWC) device for NRZ, RZand Gaussian codecs we first setup device as shown in fig1 until fig3. And same setup is repeated for one of a kind bit rate one of a kind range of the wireless channel .This optical communication system contains three predominant components, optical Transmitter, optical wireless channel and optical receiver. At transmitter a PRBS generator is used to generate records at high speed of $10 \mathrm{Gbps}$ and this feed to enter of NRZ/RZ/ Gaussian pulse generator to Generate modulating formats this electric signal in addition feed into electric enter of MZM optical modulator to with $\mathrm{CW}$ laser to modulated sign and transmitted the use of wireless verbal 
exchange channel and detected at receiver aspect the use of photo detector this signal feed into low bypass filter to eliminate excessive frequency sign which is in particular noise after this $Q$ factor and min BER analyzed the usage of BER Analyzer[7]. When I planned the models of IsOWC, which is operating at wavelength $1550 \mathrm{~nm}$ by way of the use of extraordinary modulation codec's i.e of NRZ, RZ and Gaussian pulse generator. The most modern optical wireless communication system in which the transmitter is inside the primary satellite and the receiver is within the other satellite. The unfastened area among the satellites is the propagation medium is the OWC channel this used to transmit the mild sign. Optical wireless communications use mild at close to-infrared frequency to speak. The IsOWC machine isn't an awful lot unique of unfastened area optics and fiber communication in which the distinction is based on the propagation channel[8]..

\section{A. IsOWC model utilizing $\mathrm{NRZ}$ modulation at $1550 \mathrm{~nm}$}

In this model design by using optisystem v9 software utilized the NRZ at $1550 \mathrm{~nm}$ wavelength. The power of transmitted is $10 \mathrm{dBm}$ will be utilized as shown in figure 1 .

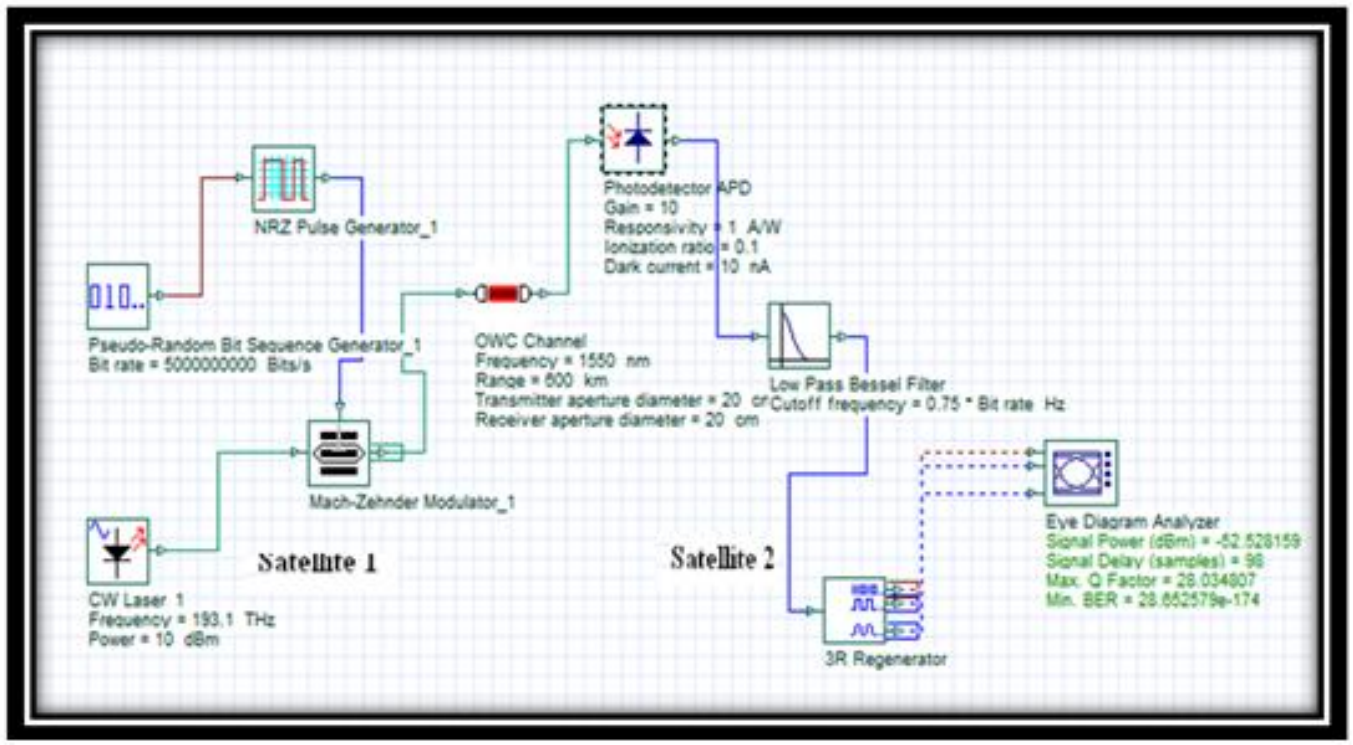

Fig.1 . IsOWC joins utilizing NRZ at wavelength $1550 \mathrm{~nm}$.

\section{B. IsOWC model utilizing RZ format at $1550 \mathrm{~nm}$}

In this model we utilized the modulation RZ at $1550 \mathrm{~nm}$ wavelength. The value of transmitting power is $10 \mathrm{dBm}$ will be utilized as shown in figure 2 .

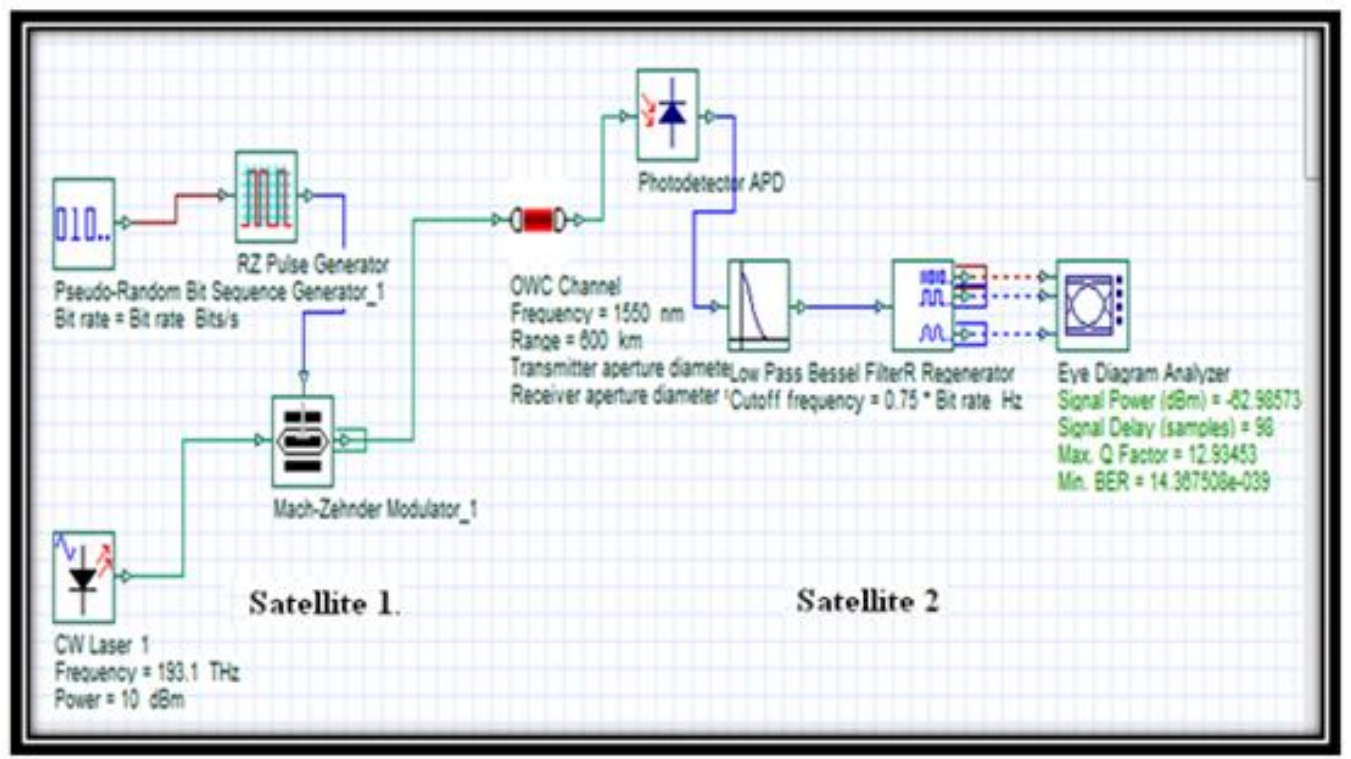

Fig.2 . IsOWC joins utilizing RZ at wavelength $1550 \mathrm{~nm}$.. 


\section{IsOWC model utilizing Gaussian pulse generator format operating at $1550 \mathrm{~nm}$}

In this model we utilized the modulation Gaussian pulse generator at $1550 \mathrm{~nm}$ wavelength. The value of transmitting power is $10 \mathrm{dBm}$ will be utilized as shown in

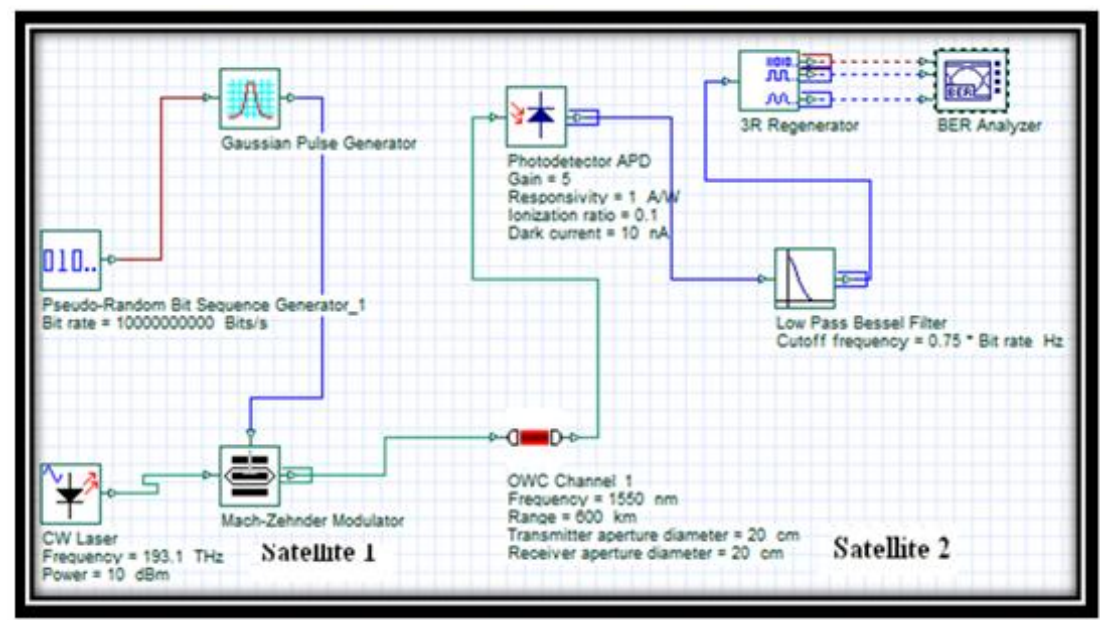

Fig.3 . IsOWC joins utilizing Gaussian at wavelength $1550 \mathrm{~nm}$..

To accomplish the IsOWC join with the maximum of selected distance $600 \mathrm{~km}$ at data bit rate $10 \mathrm{Gbps}$ and using wavelength at $1550 \mathrm{~nm}$ by using NRZ,RZ and Gaussian modulation formats. The type of laser used is $\mathrm{CW}$ laser.It can be described as the information used in shows the simulation parameter table.

Table. Simulation parameters for link at 1550nm by using NRZ ,RZ and Gaussian.

\begin{tabular}{||c|c|}
\hline Values & Parameters \\
\hline Laser & CW LASER \\
\hline Transmitting power & $10 \mathrm{dBm}$ \\
\hline $\begin{array}{c}\text { Transmission Bit } \\
\text { Rate }\end{array}$ & $10 \mathrm{Gbps}$ \\
\hline Link distance & $600 \mathrm{~km}$ \\
\hline Modulation type & NRZ,RZ,Gaussian \\
\hline Photo detector Type & $\begin{array}{l}\text { APD (Avalanche } \\
\text { Photodiode) }\end{array}$ \\
\hline Additional losses & $0.2 \mathrm{~dB}$ \\
\hline Aperture diameter of Transmitter and receiver & $20 \mathrm{~cm}$ \\
\hline Responsivity & $1 \mathrm{~A} / \mathrm{W}$ \\
\hline Dark current & $10 \mathrm{nA}$ \\
\hline
\end{tabular}

\section{RESULTS AND DISCUSSION}

In this the system distinct modulation formats have been evaluated on a single channel IsOWC link. A comparative take a look at of superior modulation schemes NRZ, RZ and Gaussian modulation formats have been developed.An the IsOWC is designed with the assist of an optisystem v9.Simulator along with two satellites with a different range of (200-400-600) km replacing outside modulated optical data rate of $10 \mathrm{Gbps}$ via the unfastened-area medium at the operating wavelength of $1550 \mathrm{~nm}$ by way of the usage of three modulation codecs i.e. NRZ, RZ and Gaussian. Above Table indicates the performance evaluation of hyperlink by means of the use of modulation codecs between two satellites . From Fig 4. Into the Fig.6.Explain measurement of the eye diagram, Q. Factor and BER by using the BER analyzer device of link in which the range is $600 \mathrm{~km}$ at the data rate is $10 \mathrm{Gbps}$ with the wavelength is $1550 \mathrm{~nm}$ by the usage of the three different modulation with transmitting and receiving diameter is $20 \mathrm{~cm}$. 


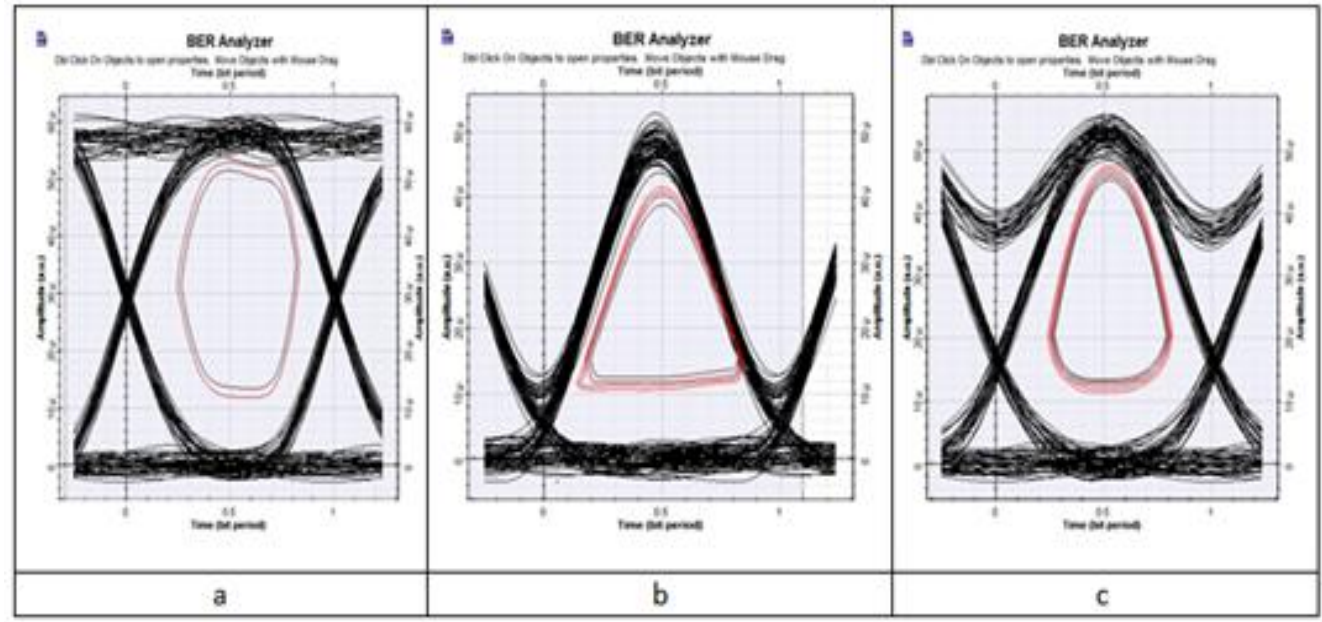

Fig. 4 :Eye diagram ofIsOWC system at range 600km, (a) NRZ Pulse Generator (b) RZ Pulse Generator .(c) Gaussian pulse generator.

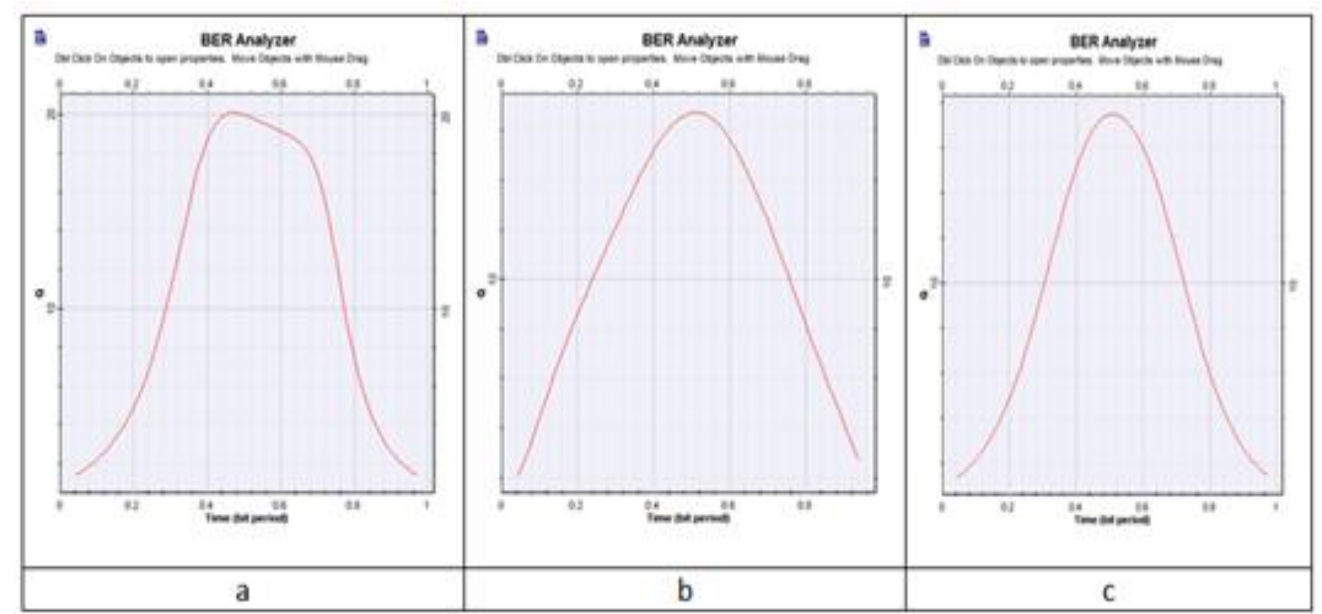

Fig.5:Maximum Q. Factor ofIsOWC system at range 600km, (a),NRZ Pulse Generator (b) RZ Pulse Generator. (c). Gaussian pulse generator.

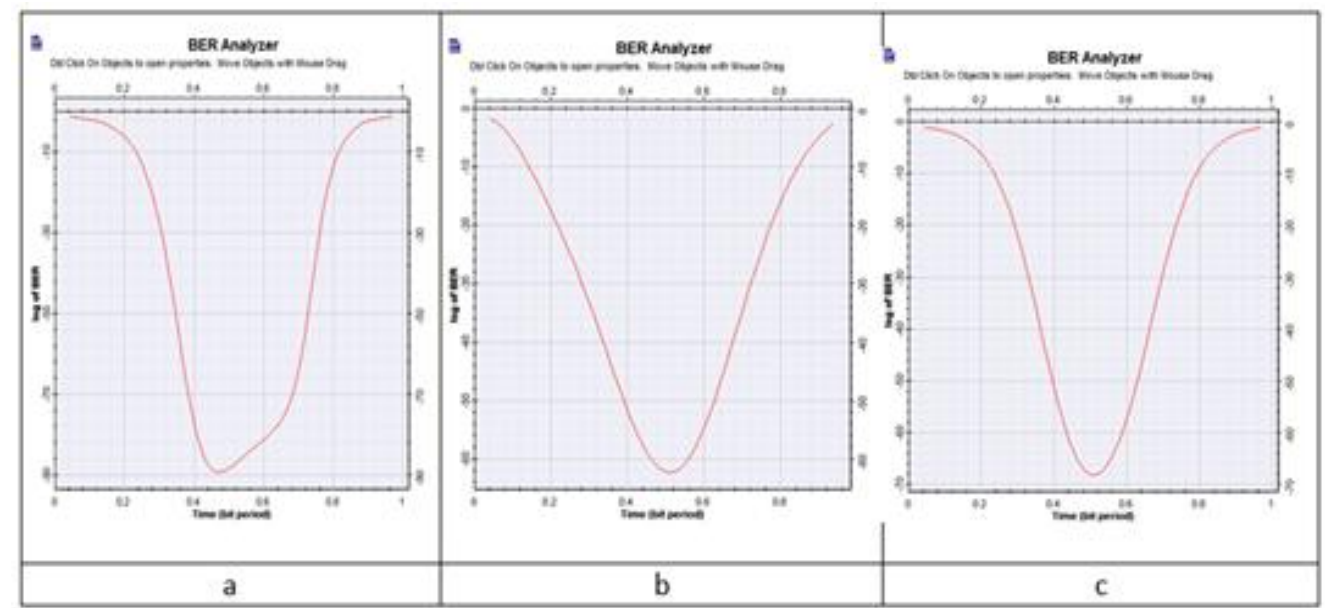

Fig.6 :Minimums BER ofIsOWC system at range 600km, (a),NRZ Pulse Generator (b) RZ Pulse Generator .(c) Gaussian pulse generator.

The relation between most extreme Q-Factor with the range, which show ended in Fig.7.This relationship, which depends on the different distance from $200 \mathrm{~km}$ in to $600 \mathrm{~km}$. The power transmitted is believed for a set value of $10 \mathrm{~dB}$. The aperture diameter is setting on $20 \mathrm{~cm}$ for three modulations of format. 


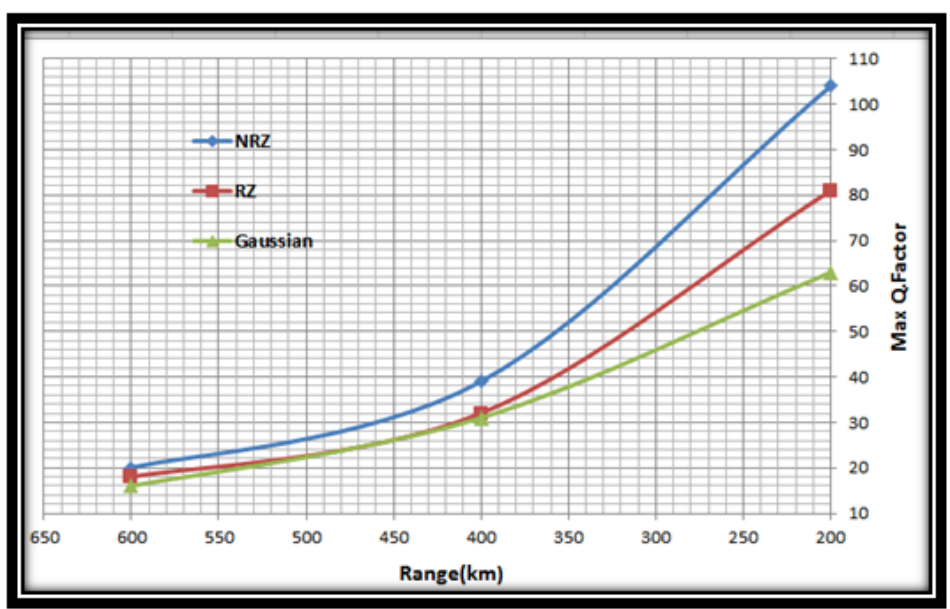

Fig7.Q-Factor vs Range for three different formats.

Fig.8 demonstrates the diagram amongst Max. Q.Factor and receiver aperture diameter, which variable from $10 \mathrm{~cm}$ into $20 \mathrm{~cm}$ at an information rate of $10 \mathrm{Gbps}$. It has been demonstrated that there is an increase in Q.Factor when increasing receiver aperture depending on the type of modulation will be used.

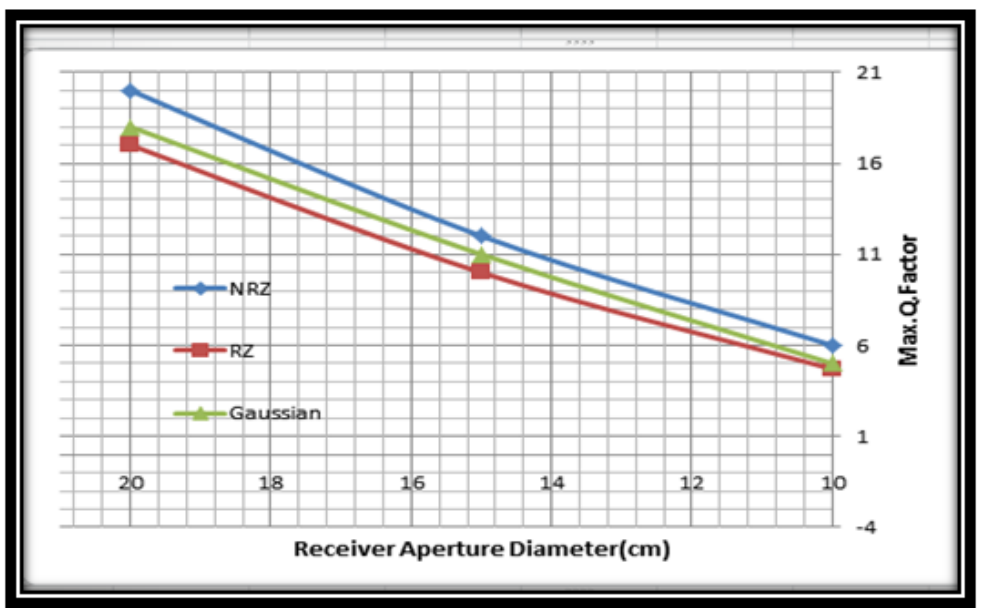

Fig.8 Q-Factor vs Receiver Aperture Diameter for three different formats.

The graphs 9 show the relationship between Min. BER and the range for different distances between two satellites at three types of modulation format.

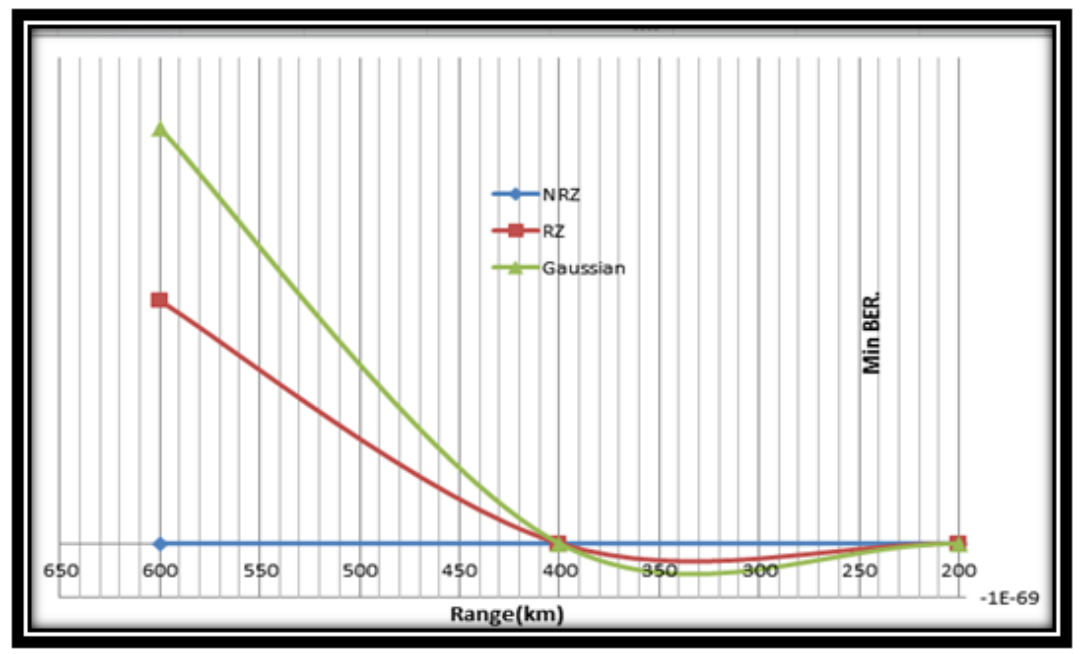

Fig.9Min.BER vs Range for three different formats 
In this paper, have a look at the overall performance of the inter satellite optical wireless conversation can be predestined in a lot ways, together with the aid of studying the Q-Factor and Min. BER. The BER we can say to be the proportion of the variance of bit mistakes disclose in the receiver and the quantity of bits transmitted, a Q-Factor is one of the vital signs of thedegree the optical overall performance through which the BER is characterized. Fig. 4 into Fig 6 explain the eye diagram of link in which the range is $600 \mathrm{~km}$ and the data rate is $10 \mathrm{Gbps}$, which operation on wavelength is $1550 \mathrm{~nm}$ with the aid of using modulation NRZ, RZ and Gauisian with transmitting energy $10 \mathrm{~dB}$., the $\mathrm{Q}$ factor is 20,18 and 17 and Min BER. Are 4.6x $10^{-90}, 4.5 \times 10^{-69}$ and $7.7 \times 10^{-69}$ respectively.Fig.7 Show the relation between maximum Q-factor with a variation of the range when compression results at $400 \mathrm{~km}$ for three format NRZ, RZ and Gaussian 39.3, 32 and 31respectively.Through these results it appeared that NRZ gives the best results.Fig.8explain the relationship of Max. Q.Factor and receiver aperture diameter, for NRZ, RZ and Gaussian format is 20, 18 and 17 respectively at receiver aperture $20 \mathrm{~cm}$.

\section{CONCLUSION}

In this work, the different modulation schemes of NRZ, RZ and Gaussian are compared with varying transmission distances and the receiver aperture diameter at 10Gbps system. The proposed gadget gave exact effects as proved from analyzing the signature course become numerous parameters of the system treats are varied. Consequently, the impactdiameters of aperture and range on system conduct is investigated. IsOWC system execution is examined in phrases of Max. Q-Factor, and Min BER.The estimation diameters of aperture across differing from $10 \mathrm{~cm}$ to $20 \mathrm{~cm}$. It's far watched that as whilst increasing the values of aperture diameters, the Q-element increases, however the bit error rate is decreasing, because inversely proportional between the QFactor and the Min.BER. But the range increased from $200 \mathrm{~km}$ into $600 \mathrm{~km}$ with a constant value of receiver aperture diameters, the value Q-factor diminished then the bit error rate is additionally expanded. Through the results we have obtained, conclude that NRZ is better than RZ and Guasain modulation. Because there is higher Q.Factor and minimum BER in NRZ rather than RZ and Guasain modulation

\section{REFERENCES}

[1]. Jump. Begley, "Free-space laser communications: a historical perspective," Annual Meeting of the IEEE, Lasers and Electro-Optics Society (LEOS) Glasgow, Scotland., Vol. 2, pp. 391-392, Nov. 2002

[2]. Jump up H. Hemmati, Deep Space Optical Communications, Wiley- Interscience, 2006

[3]. Paul Anthony Haigh, Francesco Bausi et al. "Visible light communications: real time $10 \mathrm{Mb} / \mathrm{s}$ link with a low bandwidth polymer light-emitting diode" Optics Express Vol. 22, Issue 3, 2014

[4]. Farhana Hossain, Zeenat Afroze, "Eliminating the effect of Fog attenuation on an FSO link by multiple TX/RX system with travelling wave semiconductor optical amplifier"ICAEE, Dec. 2013.

[5]. Mehtab Singh.,"Performance Evaluation of Multiple Transceiver, Optical Wireless Communication System," International Journal of Future Generation Communication and Networking Vol. 9, No. 4 (2016).

[6]. Wan Rizul, et al, "Terrestrial Free Space Optic Propagation Analysis Considering Malaysia Weather"

[7]. Navjot Kaur , Gaurav Soni " Performance analysis of inter-satellite optical wireless communication (Isaac) system by using NRZ and RZ modulation". International Journal of Scientific and Research Publications, ISSN 2250-3153, Vol..5, Issue 1, January 2015 .

[8]. Umit Kumar Pradhan, Debasish Mishra"Modeling of Inter-satellite Optical Wireless Communication (IsOWC) System"An International Journal of Engineering \& Technology, Vol. 3, No. 5 (May, 2016). 